\title{
Molecular richness of the diffuse interstellar medium: a signpost of turbulent dissipation
}

\author{
Edith Falgarone ${ }^{1}$, Benjamin Godard ${ }^{2}$, Guillaume Pineau des Forêts ${ }^{3}$ \\ and Maryvonne Gerin ${ }^{1}$ \\ ${ }^{1}$ LERMA/LRA, Ecole Normale Supérieure \& Observatoire de Paris, \\ 24 rue Lhomond, 75005 Paris, France \\ email: edith.falgarone@ens.fr \\ ${ }^{2}$ LUTh, Observatoire de Paris, 92195 Meudon, France \\ email: benjamin.godard@obspm.fr \\ ${ }^{3}$ Institut d'Astrophysique Spatiale, 91405 Orsay, France
}

\begin{abstract}
The Herschel/HIFI absorption spectroscopy surveys reveal the unexpected molecular richness of the Galactic diffuse ISM, even in gas of very low average $\mathrm{H}_{2}$ molecular fraction. In particular, two hydrides, $\mathrm{CH}^{+}$and $\mathrm{SH}^{+}$with highly endoergic formation routes have abundances that challenge models of UV-driven chemistry. The intermittent dissipation of turbulence appears as a plausible additional source of energy for the diffuse ISM chemistry. We present recent results of the so-called models of Turbulent Dissipation Regions (TDR). The abundances of many of the molecules observed in the diffuse ISM, including CO that is used as a tracer of the molecular cloud mass, may be understood in the framework of the TDR models.
\end{abstract}

Keywords. astrochemistry - turbulence - magnetic fields - ISM: molecules - ISM: kinematics and dynamics - ISM: general — ISM: evolution

\section{Introduction: the puzzles raised by the cold ISM}

The cold diffuse interstellar medium (ISM), as defined in the review of Snow \& McCall (2006), makes up the mass of nearby molecular clouds. This is best seen on the probability distribution functions (PDF) of their extinction (Kainulainen et al. 2009). Clouds devoid of star formation have log-normal PDFs up to extinctions of a few magnitudes, while actively star forming clouds have PDFs with power-law tails up to several tens of magnitude. The cloud mass is comprised in the log-normal part of the PDFs, i.e. the transparent part. Interestingly, the log-normal shape of the PDF may be seen as a signature of the multiplicative processes characteristic of turbulence.

Molecular abundances of the diffuse gas raise resilient puzzles. The first one has long been overlooked: the observed $\mathrm{CO}$ abundances in a broad range of $\mathrm{H}_{2}$ column densities exceed model predictions by more than one order of magnitude (Fig. 1 from Levrier et al. 2012, Hily-Blant \& Falgarone 2007). Another one is 70 years old. It regards the $\mathrm{CH}^{+}$abundances in the diffuse medium that exceed model predictions by two orders of magnitude. This is so because the route to $\mathrm{CH}^{+}$is highly endoergic and, once formed, $\mathrm{CH}^{+}$is rapidly destroyed by collisions with $\mathrm{H}_{2}$ to form $\mathrm{CH}_{2}^{+}$and $\mathrm{CH}_{3}^{+}$. An additional source of energy is thus required to efficiently form $\mathrm{CH}^{+}$in diffuse gas.

\section{Herschel/HIFI results}

Herschel/HIFI has even deepened these puzzles. We have conducted an absorption spectroscopy survey against bright star forming regions of the inner Galaxy (PRISMAS 


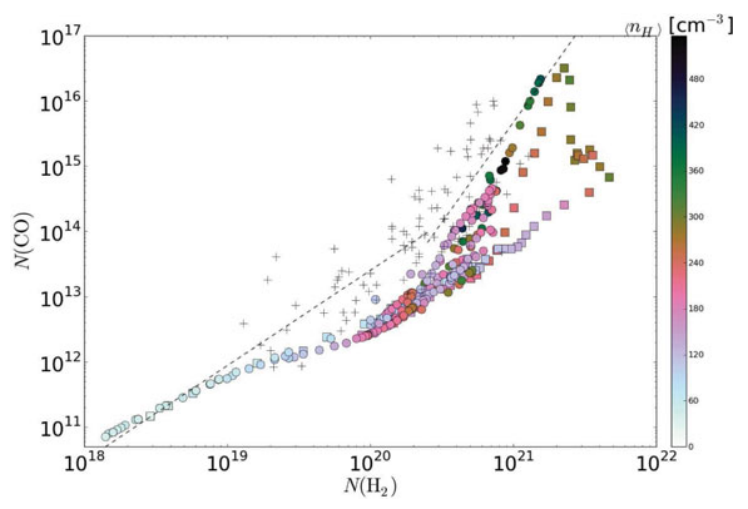

Figure 1. Comparison of observed CO column densities (crosses), derived from absorption lines against nearby stars (see references in Levrier et al. 2012) with state-of-the-art computed values combining the photo-dissociation regions (PDR) model of Le Petit et al. (2006) and bi-phasic MHD turbulence simulations of Hennebelle et al. (2008).
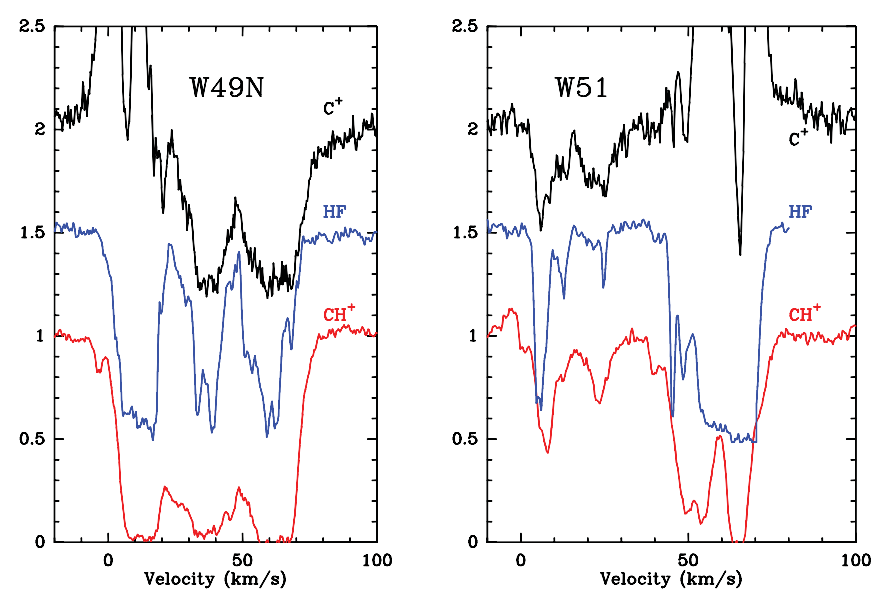

Figure 2. CII, HF and $\mathrm{CH}^{+}$spectra observed in the direction of W49 and W51. Note the similarity of the velocity coverage of the $\mathrm{CII}$ and $\mathrm{CH}^{+}$absorptions away from those of the star forming regions.

key-project, PI Gerin). Each line of sight samples kiloparsecs of gas in the Galactic plane. The results went beyond our expectations. Not only did we detect $\mathrm{CH}^{+}(1-0)$ and ${ }^{13} \mathrm{CH}^{+}$(1-0) in absorption on all the sight lines, but the $\mathrm{CH}^{+}$lines are saturated at almost all velocities (Falgarone et al. 2010a, Falgarone et al. 2010b). We also detected another hydride, $\mathrm{SH}^{+}$, that has a formation endothermicity twice as large as that of $\mathrm{CH}^{+}$(Godard et al. 2012). Moreover, $\mathrm{C}^{+}$is detected in absorption over the same velocity intervals as the $\mathrm{CH}^{+}$saturated absorption (Fig. 2). The analysis of both the CI and CII lines along the same lines of sight led us to infer that $\mathrm{C}^{+}$and $\mathrm{CH}^{+}$absorptions occur in low density gas $\left(n_{\mathrm{H}} \sim 50\right.$ to $\left.100 \mathrm{~cm}^{-3}\right)$, at temperatures $\sim 100 \mathrm{~K}$, values that are those of the cold neutral medium (CNM) (Gerin et al., in prep.). Using HF as a tracer of molecular hydrogen (Neufeld et al. 2010), and EVLA atomic hydrogen spectra (Menten et al. in prep.), we were able to assess the mean $\mathrm{H}_{2}$ molecular fraction of the absorbing gas: it is low on average and has a large scatter $0.04<f_{\mathrm{H}_{2}}<1$ among all the components (Godard et al. in prep.). Hence, $\mathrm{CH}^{+}$and $\mathrm{SH}^{+}$are detected with large abundances even in gas components with very low average $\mathrm{H}_{2}$ fractional abundance. 

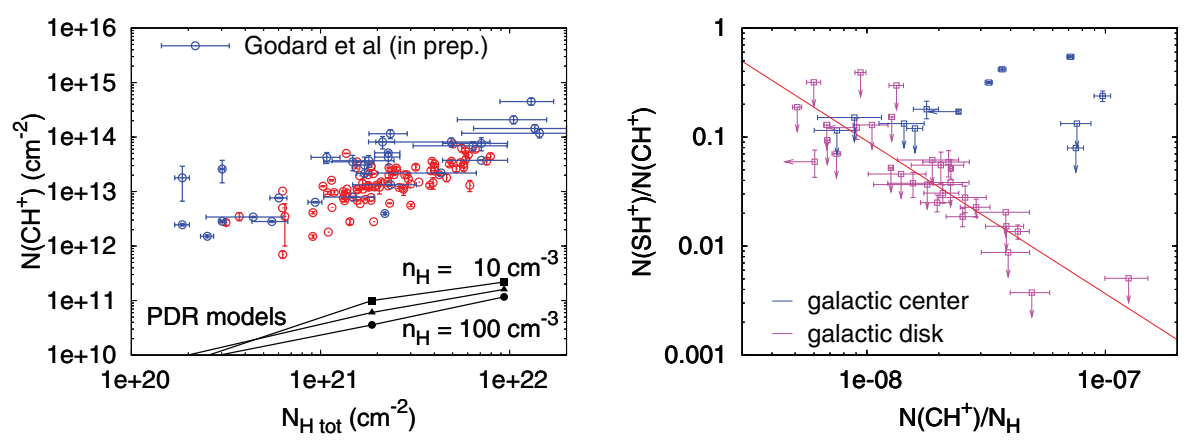

Figure 3. (Left) Observed $\mathrm{CH}^{+}$(red, visible data, blue, Herschel/HIFI data) compared to PDR model predictions. (Right) Observed $\mathrm{SH}^{+} / \mathrm{CH}^{+}$abundance ratios as a function of the $\mathrm{CH}^{+}$abundance.

UV-driven chemistry is not able either to reproduce these large $\mathrm{CH}^{+}$abundances nor the broad range of observed $\mathrm{SH}^{+} / \mathrm{CH}^{+}$ratios (Fig. 3). In PDR models, this ratio is constant and set by the slow radiative associations that initiate the formation of $\mathrm{CH}^{+}$and $\mathrm{SH}^{+}$, independently of the gas temperature. The alternative to UV-driven chemistry, is a warm chemistry that opens the route $\mathrm{C}^{+}+\mathrm{H}_{2} \rightarrow \mathrm{CH}^{+}+\mathrm{H}$ and leads to the formation of the pivotal species, $\mathrm{CH}_{3}^{+}$. In particular, $\mathrm{CH}_{3}^{+}$reacts with $\mathrm{O}$ to form $\mathrm{HCO}^{+}$, the precursor of CO. A remarkable property of $\mathrm{CH}_{3}^{+}$is that one of its photodissociation products is $\mathrm{CH}^{+}$so, unlike most molecules, $\mathrm{CH}^{+}$abundance may increase as the UV field increases (Falgarone et al. 2010a).

\section{Chemistry driven by turbulent dissipation}

Turbulence and magnetic fields that support the ISM in the gravitational well of the Galaxy (Cox 2005) are a formidable reservoir of energy. In the diffuse ISM, the bursts of turbulent dissipation are locally and temporarily a dominant source of heating for the gas. It is because the dissipation of turbulence is intermittent (see the review of Anselmet et al., 2001) that the heating rate of the gas is large enough to excite the $\mathrm{H}_{2}$ pure rotational lines by collisions (Falgarone et al. 2005, Ingalls et al. 2011) and trigger a specific "warm" chemistry. These space-time bursts are modeled as low-velocity MHD shocks (Flower \& Pineau des Forêts 1998, Lesaffre et al. 2012) and/or thin coherent vortices, (i.e. the TDR model, for Turbulent Dissipation Regions, Godard et al., 2009) temporarily heating a small fraction of the gas (a few \%) to temperatures up to $10^{3} \mathrm{~K}$. The heated gas eventually cools down once the dissipation burst is over. The free parameters of the TDR model are constrained by the known large-scale properties of turbulence. Dissipation is due to both viscosity and ion-neutral friction induced by the decoupling of the neutral fluid from the magnetic fields within the vortex. Since the diffuse medium has a low density, its chemical and thermal inertia are large. The chemical relaxation times of molecular species cover a broad range, from $200 \mathrm{yr}$ for $\mathrm{CH}^{+}$up to $5 \times 10^{4} \mathrm{yr}$ for CO. A random line of sight through the medium therefore samples 3 phases: (i) actively dissipating regions, (ii) relaxation phases, and (iii) the ambient medium.

The main successes of the TDR model are:

- the agreement of $\mathrm{CH}^{+}$and $\mathrm{SH}^{+}$observations with model predictions. An illustration is shown in Fig. 4(left). The large range of the $\mathrm{SH}^{+} / \mathrm{CH}^{+}$abundance ratio is also reproduced.

- the scaling of $\mathrm{CH}^{+}$abundances with the turbulent dissipation rate.

- the rotational excitation of $\mathrm{H}_{2}$ in diffuse gas. 

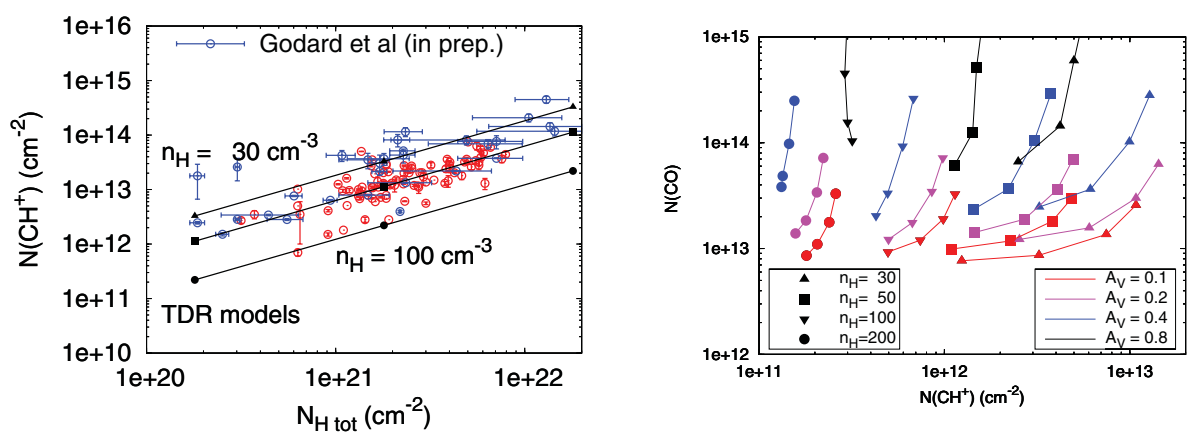

Figure 4. (Left) The $\mathrm{CH}^{+}$data compared to TDR models. (Right) $\mathrm{CO}$ and $\mathrm{CH}^{+}$column densities computed in TDR models for different densities and UV-shieldings and a total gas column density $N_{\mathrm{H}}=1.8 \times 10^{21} \mathrm{~cm}^{-2}$. The free parameter along each curve, is the rate-of-strain (Godard et al. in prep.)

- the CO abundance of diffuse molecular gas.

Fig. 4(right) displays the predicted $\mathrm{CO}$ column densities for $N_{\mathrm{H}}=1.8 \times 10^{21}$ in various models. The range of observed $\mathrm{CH}^{+}$column densities in the diffuse ISM for this total column density is $N\left(\mathrm{CH}^{+}\right)=0.2$ to $5 \times 10^{13} \mathrm{~cm}^{-2}$ (Fig. 4, left). The predicted CO columns are those observed to be far in excess to PDR predictions (see Fig. 1) in the correct range of $\mathrm{H}_{2}$ columns $\left(\sim 0.2\right.$ to $\left.\sim 1 \times 10^{21} \mathrm{~cm}^{-2}\right)$ provided by that of $f_{\mathrm{H}_{2}}$. An interesting characteristic of these models is that a fraction as small as a few percent of warm gas, heated by the dissipation of turbulent energy, is sufficient to reproduce the observed $\mathrm{H}_{2}$ line excitation diagram, as well as the abundances of specific molecules like $\mathrm{CH}^{+}$and $\mathrm{SH}^{+}$, but also CO. The data tend to be in better agreement with low rates-of-strain, i.e. models in which dissipation is dominated by ion-neutral friction.

In summary, many of the molecules we observe in the diffuse medium, including CO that is used as a tracer of the molecular mass in galaxies, are too abundant to be explained by state-of-the-art chemistry models driven by the UV-field. A plausible alternative is that they are the outcome of a specific non-equilibrium chemistry triggered by the bursts of turbulent dissipation.

\section{References}

Anselmet, F., Antonia, R., \& Danaila, L., 2001, PधSSS, 49, 1177

Cox, D., 2005, ARAESA, 43, 337

Falgarone, E., Verstraete, L., Pineau des Forêts, G., \& Hily-Blant, P., 2005, A $\& A$, 433, 997

Falgarone, E., Ossenkopf V., Gerin M. et al. 2010, A\& A, 518, L118

Falgarone, E., Godard, B., Cernicharo, J. et al. 2010, A\& A, 521, L15

Flower, D. \& Pineau des Forêts, G., 1998, MNRAS, 297, 1182

Godard, B., Falgarone, E., \& Pineau des Forêts, G., 2009, A\&A, 495, 847

Godard, B., Falgarone, E., Gerin, M., et al. 2012, A\&A, 540, 87

Hennebelle, P., Banerjee, R., Vazquez-Semadeni E. et al., 2008 A $\& A$ 486, L43

Hily-Blant, P. \& Falgarone, E., 2007, A\&A, 469, 173

Ingalls, J., Bania, T., Boulanger, F., et al. 2011, ApJ, 743, 174

Kainulainen, J., Beuther, H., Henning, T., \& Plume, R. 2009, A\&A, 508, L35

Le Petit, F., Nehmé, C., Le Bourlot, J., \& Roueff, E., 2006, ApJS, 164, 506

Lesaffre, P., Pineau des Forêts, G., Godard, B. et al., 2012, A\&A, in press

Levrier, F., Le Petit, F., Hennebelle, P., et al. A\&A, 544, 22

Neufeld, D., Sonnentrucker, P., Phillips, T., et al., 2010, A\&A, 518, L108

Snow, T. \& McCall, B., 2006, ARAESA, 44, 367 